Int. J. Electrochem. Sci., 13 (2018) 12010 - 12023

International Journal of

ELECTROCHEMICAL

SCIENCE

www.electrochemsci.org

\title{
Corrosion Protection of Water-borne Epoxy Coatings Incorporated with Graphene
}

\author{
Mingjun Cui ${ }^{1}$, Jianda Dong ${ }^{2}$, Kaihe Zhou ${ }^{2}$, Yunhui Fang ${ }^{2}$, Jibin Pu ${ }^{3}$, Haichao Zhao ${ }^{3, *}$, \\ Yonggang Wang ${ }^{1,4^{*}}$, Liping Wang ${ }^{3}$ \\ ${ }^{1}$ Key Laboratory of Impact and Safety Engineering, Ministry of Education of China, Ningbo \\ University, Ningbo 315211, China \\ ${ }^{2}$ State Grid Zhejiang Electric Power Corporation Ningbo Power Supply Company Co., Ltd, Ningbo \\ 315201, China \\ ${ }^{3}$ Key Laboratory of Marine Materials and Related Technologies, Zhejiang Key Laboratory of Marine \\ Materials and Protective Technologies, Ningbo Institute of Materials Technology and Engineering, \\ Chinese Academy of Sciences, Ningbo 315201, China \\ ${ }^{4}$ State Key Laboratory of Explosion Science and Technology, Beijing Institute of Technology, Beijing, \\ China \\ *E-mail: zhaohaichao@nimte.ac.cn, wangyonggang@ nbu.edu.cn
}

doi: $10.20964 / 2018.12 .48$

Received: 18 June 2018 / Accepted: 5 August 2018 / Published: 5 November 2018

In present study, phenosafranin (PSF) was employed to achieve the dispersion of graphene in water owing to the presence of $\pi-\pi$ interaction which was also confirmed from the absorption free energy calculated by first principles based on density functional theory. In addition, water-borne epoxy (WEP) coatings containing graphene, PSF and PSF@G were prepared and fabricated on the electrodes. Meanwhile, the corrosion protection performance of different coatings during the immersion in 3.5 wt $\% \mathrm{NaCl}$ solution was investigated and compared. It could be concluded from the results that PSF@G/WEP coating presented better protective performance than other coatings since the well dispersed graphene in WEP provided superior impermeability to corrosive medium. Besides, the PSF surfactant used to disperse graphene was also beneficial to improve the protective performance of the WEP coating.

Keywords: Graphene, Phenosafranin, $\pi-\pi$ interaction, Water-borne epoxy, Corrosion protection

\section{FULL TEXT}

(C) 2018 The Authors. Published by ESG (www.electrochemsci.org). This article is an open access article distributed under the terms and conditions of the Creative Commons Attribution license (http://creativecommons.org/licenses/by/4.0/). 